\title{
Énoncé sur l'aménagement des territoires au Québec
}

\author{
Association des aménagistes régionaux du Québec
}

$\int$ es 20 et 21 octobre 2004, l'Association des aménagistes régionaux du Québec (AARQ) tenait un important congrès portant principalement sur une rétrospective des deux décennies de pratique de l'aménagement du territoire au Québec. Rétrospective effectuée pertinemment dans un esprit de questionnement de cette pratique professionnelle concernée par l'ajout de rationalité dans les modalités d'occupation et de développement du territoire.

L'aménagement des territoires du Québec existe évidemment depuis très longtemps, autant dans l'action directe par la construction d'équipements et d'infrastructures que par la planification de ces actions qu'on appelle souvent pratique de l'aménagement. $\mathrm{Au}$ Québec, la construction de ports, bâtiments,

\section{Le congrès de l'AARQ désirait débattre des leçons distillées de cette importante expérience, notamment les acquis actuels.}

le grand thème de cet important événement de réflexion collective.

L'énoncé qui est présenté dans ce texte fut rédigé à partir des notes recueillies au cours des débats effectués à l'intérieur de quatre ateliers animés autour de quatre grands thèmes. Il s'agit de l'aménagement du territoire comme tel, des acteurs de cette pratique professionnelle, de l'organisation et du mandat des entités MRC ainsi que de la mise en œuvre des schémas d'aménagement et de développement et de leur financement. Pour les participants, chacun des thèmes était illustré par une courte problématique afin d'offrir une mise en situation commune bien ciblée sur les enjeux pertinents. Des constats et des questions furent posés clairement. Les débats ont ainsi été enclenchemins, canaux remontent déjà à quelques siècles, alors qu'un aménagement extensif des territoires eut lieu au cours du $\mathrm{XX}^{\mathrm{e}}$ siècle. Depuis la mise en vigueur de la Loi sur l'aménagement et l'urbanisme au début des années 1980, les territoires MRC (municipalités régionales de comté) et les communautés métropolitaines sont désormais tenus d'utiliser formellement une procédure de planification bien définie, qui conduit les collectivités à la confection et à l'application de schémas d'aménagement et de développement. Deux décennies de pratique professionnelle qui ont sollicité non seulement les aménagistes mais aussi, à degrés variables, les élus municipaux, les différents acteurs (scolaire, transport, agriculture, industrie...) ainsi que les divers groupes de la société civile (loisirs, chambres de commerce, communautaire....). Le congrès de l'AARQ désirait débattre des leçons distillées de cette importante expérience, notamment les acquis actuels. Des universitaires et des philosophes furent aussi sollicités par ce congrès. Dans la foulée, les aménagistes régionaux réunis désiraient ouvrir et alimenter le débat sur les perspectives d'avenir. «20 ans d'aménagement... et après ?», tel était chés à un très haut niveau. Les résumés effectués représentent une riche source documentaire. Plusieurs propositions et recommandations furent formulées. À l'analyse de leur convergence, l'énoncé qui suit fut rédigé autour de six propositions principales.

\section{Principes de base encore valables}

Dès le départ, l'AARQ avance que les quatre grands principes de la loi de 1979 sont encore tout à fait valables en 2005, même s'ils ne sont pas très simples à appliquer, notamment la mobilisation des citoyens. À l'article 19.1 de la LAU, ces principes sont formulés ainsi :

1. l'aménagement est d'abord une responsabilité politique;

2. le citoyen est associé aux diverses étapes du processus;

3. l'aménagement est une fonction partagée entre les trois paliers de décisions : municipalité, MRC et gouvernement; 
4. l'aménagement fait appel à la coordination et à la conciliation des choix et aux trois paliers de décisions.

Les aménagistes considèrent l'énorme évolution effectuée en pratique de l'aménagement du territoire depuis vingt ans, soit depuis la première génération de schémas adoptés par les MRC. La culture de la planification s'est certes enrichie chez les élus locaux, notamment grâce à la montée des préoccupations environnementales bien exprimées. Aussi, la dimension concernée par le développement économique, qui ne fut pas prévue initialement par la loi, fut intégrée tant bien que mal au processus de planification territoriale, notamment pendant la deuxième génération des schémas.

Par ailleurs, on s'entend en général pour considérer la lourdeur de fonctionnement autant dans le processus décisionnel des conseils MRC que dans la procédure de planification territoriale qui est imposée par la loi. Les aménagistes régionaux du Québec n'hésitent pas à recommander leur allègement afin de gagner de la rapidité et de l'efficacité.

\section{Préséance de la LAU}

Les acteurs et les intervenants dans la pratique de l'aménagement des territoires au Québec sont nombreux, notamment les divers ministères des gouvernements supérieurs, les sociétés d'État, les municipalités locales et les entreprises du secteur privé. Même avec des instruments de planification tels que les schémas d'aménagement et de développement des MRC et des communautés métropolitaines, il apparaît difficile de coordonner leurs interventions selon des orientations déterminées. À l'heure où l'exercice de révision $\left(2^{\mathrm{e}}\right.$ génération) des schémas d'aménagement et de développement est toujours en voie d'être terminé, les aménagistes régionaux du Québec s'interrogent sur l'efficacité réelle des instruments mis à la disposition de la population et de leurs élus pour orienter les actions qui ont une incidence sur l'aménagement des territoires. Ils constatent que la Loi sur l'aménagement et l'urbanisme (LAU) n'a pas ou peu préséance sur les initiatives sectorielles diverses, notamment celles du gouvernement du Québec.

À cet effet, les aménagistes proposent la création d'une instance supérieure de planification spatiale au
Québec. Il pourrait s'agir d'un ministère d'État à l'Aménagement et au Développement des territoires, d'un Secrétariat bien mandaté au sein du Conseil exécutif ou encore d'une Commission permanente pour l'aménagement. Quel que soit le mécanisme intersectoriel retenu à travers les options préconisées, l'AARQ propose d'abord de réaffirmer clairement la finalité supérieure de la planification spatiale au Québec. Des états généraux de l'aménagement pourraient être réalisés au Québec en mobilisant les divers intérêts «territoriaux », notamment l'environnement, le paysage, l'énergie, la forêt, l'eau, le développement durable et ce, au printemps 2006.

En outre, l'AARQ suggère au gouvernement de renouveler adéquatement sa capacité d'agir globalement par l'entremise de la révision de la LAU, laquelle doit s'adapter adéquatement à la conjoncture contemporaine afin de bien servir les grandes orientations dans l'utilisation des territoires au Québec. Signalons, à cet effet d'adaptation, la montée en puissance des préoccupations sociales à l'égard de l'environnement qui rend désormais tout à fait incontournable la finalité du développement durable.

\section{On s'entend en général pour considérer la lourdeur de fonctionnement autant dans le processus décisionnel des conseils MRC que dans la procédure de planification territoriale qui est imposée par la loi.}

\section{Vision globale des territoires}

L'affirmation de la volonté gouvernementale pour une nouvelle régulation en matière d'aménagement des territoires devrait s'inscrire au préalable, selon l'AARQ, par l'élaboration d'une vision globale des territoires qui composent le vaste espace qu'est le Québec. Un tel exercice nous réfère bien sûr à une réflexion profonde et sérieuse sur les valeurs, les buts et les finalités devant conduire à des choix territoriaux de société à enchâsser dans la LAU. Il convie aussi à la prise en compte explicite et détaillée de la réalité par l'entremise de la confection d'un portrait complet de la situation des divers territoires au Québec. Les travaux de la récente commission Coulombe sur la forêt boréale se sont parfaitement inscrits dans ce double sens du "visionning », tout en demeurant certes 
limités à un secteur d'activité et à une composante territoriale, soit la boréalie. Globalisée systématiquement dans un esprit d'ouverture à la nouvelle connaissance et aux nouvelles finalités sociétales, une telle vision élaborée pourrait devenir le préambule de la LAU révisée. Préambule dans lequel serait présentées, selon l'AARQ, la mission nationale de planification spatiale, l'importance du développement durable, la nécessaire implication des élus ainsi que la responsabilité collective au Québec de l'aménagement des territoires qui composent le vaste espace.

\section{Cette dynamique territoriale « MRC » illustre aujourd'hui une assez grande diversité d'arrangements institutionnels.}

Mis à part les différentes aires de gestion découpées telles que les régions administratives, les MRC, les municipalités, les arrondissements, les comtés, les commissions scolaires, etc., les composantes territoriales du Québec font aussi référence aux territorialités des phénomènes économiques. À cet effet de composantes territoriales de nature économique, soulignons notamment les diverses zones découpées pour l'agriculture, l'industrie, le commerce, l'eau, l'exploitation de la forêt, des mines, de la pêche.

En considérant leurs spécificités sous l'angle des contenus, ces zones spécialisées d'activités économiques épousent aussi des formes dynamiques mouvantes au fil du temps. Les tendances spatiales doivent à cet égard être prises en compte avec les meilleurs outils de mesure disponibles. Seraient alors illustrés les phénomènes de métropolisation, de concentration, d'exode, de dévitalisation, de dispersion. En réalité, les zones spécialisées d'activités économiques se forment et se déforment en réponse à des forces contemporaines particulières. Si la force spatiale de la gravité qui caractérise Toronto s'avère bien connue à cet effet, d'autres agissent considérablement telles que l'explosion actuelle des échanges nord-sud, l'intégration économique sectorielle, la croissante mobilité des travailleurs, des consommateurs et des marchandises, l'attractivité de bassins nordiques de ressources, l'émergence des collectivités autochtones, l'appropriation territoriale de leviers économiques. Bref, l'élaboration de la vision globale de l'espace au Québec implique non seulement d'élaborer une clas- sification des territoires en fonction de certains critères et de certaines caractéristiques relativement statiques, mais nécessite aussi de considérer l'aspect dynamique. La saisie des contenus, des formes, des forces et des tendances de cette dynamique spatiale québécoise n'est certes pas évidente, mais elle s'avère d'une grande pertinence sociale pour offrir un cadre de référence à la volonté gouvernementale d'aménager les divers territoires d'une manière rationnelle.

\section{Le renforcement de la structure MRC}

Mises en place depuis plus de deux décennies, les MRC furent structurées au fil du temps par des fonctions publiques (aménagement et développement, matières résiduelles, sécurité civile...) sur des territoires bien découpés sur lesquels les représentants des municipalités locales forment le conseil (de la MRC). Ces dernières furent parallèlement l'objet d'une construction institutionnelle par diverses organisations publiques et collectives. Soulignons ici certaines commissions scolaires, un grand nombre de centres et de sociétés (CLSC, CLE, SADC, CLD...) ainsi que de multiples groupes issus de la société civile. Cette dynamique territoriale « MRC » illustre aujourd'hui une assez grande diversité d'arrangements institutionnels se situant de la faible présence de la corporation principale jusqu'à la communauté bien consolidée, en passant par la MRC coopérative de services. Une analyse fine des quelque 90 territoires MRC fait apparaître une très grande variété de statuts plus ou moins formels qui fait d'ailleurs la richesse et la force de ces territoires. L'appartenance territoriale à une MRC est un acquis institutionnel certes multiforme et variable, mais qui permet désormais d'asseoir un renforcement bien ciblé du potentiel d'appropriation territoriale.

Acteurs privilégiés de cette évolution des territoires de MRC, les aménagistes régionaux du Québec préconisent le renforcement de leurs pouvoirs. Ils font consensus autour de la nécessité d'avoir des règles du jeu claires et cohérentes afin de consolider le rôle et le mandat du conseil de la MRC. À cet effet, l'AARQ recommande de favoriser l'élection au suffrage universel du préfet de la MRC ainsi que son engagement à temps plein. L'exemple des villes-MRC en opération actuellement illustre des processus décisionnels plus légers, plus flexibles et plus efficaces dont les MRC ont tout intérêt à s'inspirer. 


\section{Le schéma d'aménagement et de développement comme pivot de cohésion territoriale}

Si l'ajout éventuel de nouvelles responsabilités publiques, de nouveaux pouvoirs et de nouveaux mandats s'avère une option acceptable et faisable pour les MRC, l'AARQ considère qu'il faut surtout recentrer la mission principale de ces organisations municipales autour de l'axe central du mandat relié à l'aménagement et au développement du territoire. Les autres fonctions secondaires doivent se greffer autour, comme des branches à un tronc d'arbre, dans un esprit de renforcement de la cohésion et de la cohérence globale. Car la confection et la mise en œuvre du schéma d'aménagement et de développement du territoire pourraient devenir le véhicule du renforcement de l'appropriation territoriale de fonctions publiques et de leviers économiques. En réalité, l'élaboration d'une vision commune du territoire par l'ensemble des acteurs d'une MRC possède, selon l'expérience acquise des aménagistes, la vertu d'intégration et de consolidation entre les divers planificateurs présents. Le schéma d'aménagement et de développement à contenu variable selon les territoires peut potentiellement s'inscrire tel un projet collectif, indicatif mais aussi coercitif et incitatif. Dans son contenu défensif actuel, le schéma possède tout le potentiel pour devenir offensif et proactif.

À cet effet, la procédure de planification à utiliser s'avère très importante. Forts de leur expérience, les aménagistes régionaux préconisent de raccourcir et d'alléger la procédure prévue par la LAU jadis utilisée à deux reprises. Celle-ci doit être plus flexible, plus mobilisatrice des divers acteurs et surtout plus inclusive de la dimension concernée par le développement. La multiplication des petites tribunes et des petits événements pour soutenir le dialogue, la concertation et l'apprentissage collectif apparaît, selon l'AARQ, une formule à explorer pour rendre la procédure de planification territoriale plus interactive et aussi plus inclusive des forces locales associées au développement endogène. Grâce à cette interaction mobilisatrice d'expertises et en principe créatrice d'initiatives, le schéma d'aménagement et de développement sera revalorisé et repositionné dans son rôle de pivot central de l'intégration territoriale des actions et interventions. À terme, il permettra d'harmoniser à degré élevé le contenu des plans sectoriels

conçus à l'échelle des MRC et aussi à l'échelle des régions administratives.

\section{Forts de leur expérience, les aménagistes régionaux préconisent de raccourcir et d'alléger la procédure prévue par la LAU jadis utilisée à deux reprises.}

\section{Le financement autonome}

Dans le contexte actuel de la LAU, la mise en œuvre du schéma d'aménagement et de développement passe par les plans d'urbanisme des municipalités et par les autres plans locaux ou sectoriels. Aussi, les actions des individus et des entreprises sont encadrées et régulées par les orientations stratégiques contenues dans les schémas; orientations qui possèdent un statut légal afin d'être appliquées convenablement sur le terrain. Bien qu'elles possèdent de nombreuses vertus, ces modalités de planification territoriale de nature défensive ne sont guère proactives ou offensives.

Après deux décennies d'expérimentation de cette formule de planification offerte par la LAU, les aménagistes régionaux du Québec recommandent la dotation au planificateur d'une véritable capacité financière spécifique pour la mise en œuvre des schémas. Il s'agit d'une condition sine qua non à la proactivité. Une enveloppe territoriale rattachée au conseil de la MRC devrait être dédiée spécifiquement pour le montage de projets d'aménagement. Une fois la faisabilité validée, les actions concrètes pourraient ainsi cheminer beaucoup plus rapidement auprès des partenaires financiers de leur réalisation. En réalité, la dotation d'une enveloppe spécifique de financement dédiée à la faisabilité technique, économique, environnementale, financière, sociale de projets d'aménagement et de développement pour chaque territoire de MRC jouerait le rôle de passerelle effective entre le cadre stratégique offert par le schéma d'aménagement et de développement et les actions concrètes disjointes dans le milieu.

Plusieurs formules peuvent être envisagées à cet égard de financement autonome, notamment une dotation par territoire de MRC sur la base de ratios. Le récent pacte rural offre une marge de manœuvre financière pour Québec, alors que la taxe de vente demeure une voie intéressante à explorer par les instances supérieu- 
res. Aussi, les redevances perçues par Québec sur les droits d'exploitation des ressources naturelles pourraient être redistribuées sur les territoires en utilisant notamment un mécanisme de péréquation. Nous pouvons penser aussi au transfert de l'impôt scolaire aux MRC ou à l'établissement d'un impôt foncier à cette échelle territoriale. Bref, il existe quelques formules à analyser afin de doter les conseils de MRC d'une autonomie financière suffisante pour stimuler l'émergence d'action dans le cadre de l'application de leur schéma d'aménagement et de développement respectif.

L'AARQ propose à la LAU une audacieuse avancée pouvant soutenir les élus des MRC et les acteurs sur les territoires vers une meilleure appropriation de leurs responsabilités et de leurs leviers économiques.

\section{Conclusion}

En retard sous l'angle du contrôle de l'utilisation du sol, mais paradoxalement précurseur en matière de préoccupation environnementale, le gouvernement du Québec s'est doté en 1979 d'une loi originale sur l'aménagement et l'urbanisme. Vingt ans après le coup d'envoi de la confection de la première génération de schémas, les aménagistes régionaux regroupés au sein de l'AARQ ont eu le courage d'effectuer une ré- flexion collective afin de tirer des leçons et regarder vers l'avenir. Cette réflexivité s'avère essentielle au progrès collectif. Du coup, ils ont élaboré des propositions qui s'inscrivent largement dans l'énoncé formulé dans ces pages.

Deux dimensions nouvelles de l'aménagement des territoires semblent désormais incontournables pour les aménagistes régionaux, soit les préoccupations environnementales et le développement territorial. Elles ne sont pas exclusives. Et leur prise en compte, selon l'AARQ, doit ouvrir la procédure de planification territoriale vers les différents acteurs et planificateurs sectoriels. En réalité, l'AARQ propose à la LAU une audacieuse avancée pouvant soutenir les élus des MRC et les acteurs sur les territoires vers une meilleure appropriation de leurs responsabilités et de leurs leviers économiques. Dans cette offre formulée en énoncé, Québec devrait y voir une source de progrès territorial proposé par des experts qui observent et agissent sur la scène des MRC depuis vingt ans.

Le comité organisateur $d u 20^{e}$ anniversaire de l'AARQ,

Érick Olivier, président (MRC Nouvelle-Beauce)

Serge Bougeois (municipalité des Iles-de-la-Madeleine)

Isabelle Lessard (MRC de Maskinongé)

André Boisvert (MRC des Pays d'en haut)

Nathalie Audet (MRC de Lac-Saint-Jean-Est) 


\section{Publicité}

«Doctorat en développement régional » 